\title{
INTERPLANETARY TYPE III BURSTS AND DENSITY FLUCTUATIONS IN THE SOLAR WIND
}

\author{
V. Krupar*, O. Santolik*, J. Soucek*, O. Kruparova*, M. Maksimovic ${ }^{\dagger}$, \\ E. Kontar ${ }^{\ddagger}$, and J. Eastwood ${ }^{\S}$
}

\begin{abstract}
Type III bursts are generated by beams of fast electrons originated from reconnection sites of solar flares. These beams propagate outwards from the Sun along open magnetic field lines in the corona and in the interplanetary (IP) medium while exciting radio emission at the local plasma frequency $f_{p}$ and/or its second harmonic $2 f_{p}$. We performed a statistical survey of 152 simple and isolated IP type III bursts observed by STEREO/Waves instruments between May 2007 and February 2013. We investigated their time-frequency profiles in order to derive decay times as a function of frequency. Next, we performed Monte Carlo simulations to study a role of scattering due to random density fluctuations on time-frequency profiles of radio emissions generated in the IP medium. Derived decay times from observations and simulations were compared. We conclude that relative electron density fluctuations $\left\langle\delta n_{e}\right\rangle / n_{e}$ at the outer scale of turbulence in the solar wind are 0.15 and 0.30 assuming the fundamental and harmonic emission, respectively.
\end{abstract}

\footnotetext{
* Department of Space Physics, Institute of Atmospheric Physics, Czech Academy of Sciences, Prague, Czech Republic

$\dagger$ LESIA, Observatoire de Paris, Meudon, France

$\ddagger$ University of Glasgow, Glasgow, UK

§Imperial College London, London, UK
} 
